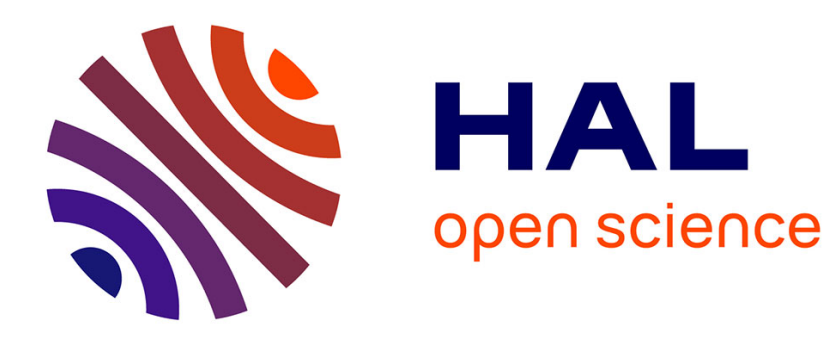

\title{
Conjectures for large transcendence degree
}

Michel Waldschmidt

\section{To cite this version:}

Michel Waldschmidt. Conjectures for large transcendence degree. Number Theory Conference Graz, Aug 1998, Graz, Austria. pp.497-520. hal-00416164

\section{HAL Id: hal-00416164 https://hal.science/hal-00416164}

Submitted on 12 Sep 2009

HAL is a multi-disciplinary open access archive for the deposit and dissemination of scientific research documents, whether they are published or not. The documents may come from teaching and research institutions in France or abroad, or from public or private research centers.
L'archive ouverte pluridisciplinaire HAL, est destinée au dépôt et à la diffusion de documents scientifiques de niveau recherche, publiés ou non, émanant des établissements d'enseignement et de recherche français ou étrangers, des laboratoires publics ou privés. 


\title{
Conjectures for Large Transcendence Degree
}

\author{
Michel Waldschmidt
}

\begin{abstract}
We state two conjectures, which together would yield strong results of algebraic independence related to Schanuel's Conjecture. Partial results on these conjectures are also discussed.
\end{abstract}

1991 Mathematics Subject Classification: 11J82, 11J85.

\section{Introduction}

Connections between algebraic independence and Diophantine approximations arose recently [19], [20], [9], [10], [11], [14], [17]. Several survey papers have already been devoted to this subject [7], [24], [25] (see also [26] Chap. 17). The purpose of the present article is to give heuristics for algebraic independence. More precisely we state two main conjectures. The first one (Conjecture 1) relates the transcendence degree of a field (finitely generated over $\mathbb{Q}$ ) with Diophantine approximation properties of a transcendence basis. The second one (Conjecture 2) is an estimate from below for the distance between some transcendental numbers and algebraic numbers. Usually, in such estimates, the main parameter measures the height of the approximating algebraic numbers, but here the leading role is played by the degree.

Combining these two conjectures, one deduces strong results of algebraic independence related to Schanuel's Conjecture ([5] Chap. III, Historical Note p. 30-31; [3] p. 260).

Schanuel's Conjecture. Let $x_{1}, \ldots, x_{n}$ be $\mathbb{Q}$-linearly independent complex numbers. Then, among the $2 n$ numbers

$$
x_{1}, \ldots, x_{n}, e^{x_{1}}, \ldots, e^{x_{n}},
$$

at least $n$ are algebraically independent.

For instance our two main conjectures would solve the following problem of algebraic independence of logarithms of algebraic numbers. 
Conjecture on Independence of Logarithms of Algebraic Numbers. If logarithms of algebraic numbers $\lambda_{1}, \ldots, \lambda_{n}$ are linearly independent over $\mathbb{Q}$, then they are algebraically independent over $\mathbb{Q}$.

Also one would be able to deduce the algebraic independence of numbers like $e, \pi, e^{\pi}, e^{\pi^{2}} \ldots$

Our approach enables us to compare the difficulty of different open problems.

Finally we propose further conjectures (which are likely to be easier ones) related to almost all complex uples.

\section{Connections Between Transcendence Degree and Diophantine Estimates}

Our first main conjecture relates the transcendence degree of the field generated by a $m$-uple $\left(\theta_{1}, \ldots, \theta_{m}\right)$ of complex numbers with Diophantine approximation properties of this $m$-uple (see [7], Conjectures 5 and 5'; compare with [20], Conjecture 1.7; [14], Problems 7 and 8 p. 317, Problem 10 p. 319; [24], Conjecture 2.4; [25], Conjecture 17 and [18], Conjecture).

For an algebraic number $\gamma$ of degree $d$ and minimal polynomial

$$
a_{0} X^{d}+\cdots+a_{d}=a_{0}\left(X-\gamma^{(1)}\right) \cdots\left(X-\gamma^{(d)}\right) \in \mathbb{Z}[X],
$$

we define the absolute logarithmic height $\mathrm{h}(\gamma)$ by

$$
\mathrm{h}(\gamma)=\frac{1}{d} \log \mathrm{M}(\gamma) \quad \text { where } \quad \mathrm{M}(\gamma)=a_{0} \prod_{i=1}^{d} \max \left\{1,\left|\gamma^{(i)}\right|\right\}
$$

Estimates relating $\mathrm{h}$ with the so-called usual height

$$
\mathrm{H}(\gamma)=\max _{0 \leq i \leq d}\left|a_{i}\right|
$$

can be deduced from

$$
2^{-d} H(\gamma) \leq \mathrm{M}(\gamma) \leq H(\gamma) \sqrt{d+1}
$$

(See for instance Chapter 3 of [26]).

For a $m$-uple $\underline{\gamma}=\left(\gamma_{1}, \ldots, \gamma_{m}\right)$ of algebraic numbers, we define

$$
\mu(\underline{\gamma})=[\mathbb{Q}(\underline{\gamma}): \mathbb{Q}] \max _{1 \leq j \leq m} \mathrm{~h}\left(\gamma_{j}\right)
$$

so that for $m=1$ and $\gamma \in \overline{\mathbb{Q}}$ we have simply $\mu(\gamma)=\log \mathrm{M}(\gamma)$.

Conjecture 1. Let $\underline{\theta}=\left(\theta_{1}, \ldots, \theta_{m}\right)$ be a m-uple of complex numbers, not all of which are algebraic, so that the transcendence degree

$$
t=\operatorname{trdeg}_{\mathbb{Q}} \mathbb{Q}(\underline{\theta})
$$


over $\mathbb{Q}$ of the field $\mathbb{Q}\left(\theta_{1}, \ldots, \theta_{m}\right)$ is $\geq 1$. There exist positive constants $c_{1}$ and $c_{2}$ with the following property. Let $\left(D_{\nu}\right)_{\nu \geq 0}$ and $\left(\mu_{\nu}\right)_{\nu \geq 0}$ be sequences of real numbers satisfying

$$
c_{1} \leq D_{\nu} \leq \mu_{\nu}, \quad D_{\nu} \leq D_{\nu+1} \leq 2 D_{\nu}, \quad \mu_{\nu} \leq \mu_{\nu+1} \leq 2 \mu_{\nu} \quad(\nu \geq 0) .
$$

Assume also that the sequence $\left(\mu_{\nu}\right)_{\nu \geq 0}$ is unbounded. Then for infinitely many $\nu$ there exists a m-uple $\left(\gamma_{1}, \ldots, \gamma_{m}\right)$ of algebraic numbers satisfying

$$
[\mathbb{Q}(\underline{\gamma}): \mathbb{Q}] \leq D_{\nu}, \quad \mu(\underline{\gamma}) \leq \mu_{\nu}
$$

and

$$
\max _{1 \leq i \leq m}\left|\theta_{i}-\gamma_{i}\right| \leq \exp \left\{-c_{2} D_{\nu}^{1 / t} \mu_{\nu}\right\}
$$

Remark 1. Conjecture 1 is known for $t=1$ (cf. Proposition 2 in $\S 5.2$ below).

Remark 2. It is shown in [4] p. 180 that a criterion for algebraic independence of Philippon ([3] Chap. $6 \S 3.2 ;$ [24] Th. 2.2; [13]) follows from Conjecture 1.

Remark 3. As shown by D. Roy [18], Conjecture 1 is sharp: a lower bound for the degree $[\mathbb{Q}(\gamma): \mathbb{Q}]$ cannot be required, and one cannot replace the condition $\mu(\underline{\gamma}) \leq \mu_{\nu}$ by the weaker one

$$
\max _{1 \leq i \leq m} \mathrm{~h}\left(\gamma_{i}\right) \leq \frac{\mu_{\nu}}{D_{\nu}} .
$$

Remark 4. A heuristic motivation is given by M. Laurent in [7] $\S 4.2$ p. 325 .

Definition. Let $\theta_{1}, \ldots, \theta_{m}$ be complex numbers. A function

$$
\psi: \mathbb{N} \times \mathbb{R}_{>0} \rightarrow \mathbb{R}_{>0} \cup\{\infty\}
$$

is a simultaneous approximation measure for $\theta_{1}, \ldots, \theta_{m}$ if there exists a positive integer $D_{0}$ such that, for any integer $D \geq D_{0}$, any real number $\mu \geq D$ and any $m$-uple $\left(\gamma_{1}, \ldots, \gamma_{m}\right)$ of algebraic numbers satisfying

$$
[\mathbb{Q}(\underline{\gamma}): \mathbb{Q}] \leq D \quad \text { and } \quad \mu(\underline{\gamma}) \leq \mu
$$

we have

$$
\max _{1 \leq i \leq m}\left|\theta_{i}-\gamma_{i}\right| \geq \exp \{-\psi(D, \mu)\}
$$

Remark 1. A measure is "sharp" if $\psi$ is "small".

Remark 2. The corresponding definition in [20] is phrased in terms of $D$ and $h=\max h_{i}$. Roughly speaking, the function $\varphi(D, h)$ of [20] is related with our $\psi(D, D h)$, but there is a discrepancy: if one sets $\mu=D h$, one should beware that the conditions

$$
[\mathbb{Q}(\underline{\gamma}): \mathbb{Q}] \leq D, \quad \mu(\underline{\gamma}) \leq \mu
$$


and

$$
[\mathbb{Q}(\underline{\gamma}): \mathbb{Q}] \leq D, \quad \max _{1 \leq i \leq m} \mathrm{~h}\left(\gamma_{i}\right) \leq h
$$

do not coincide. Our change of definition is motivated by [18].

A simultaneous approximation measure for $\theta_{1}, \ldots, \theta_{m}$ is obviously also a simultaneous approximation measure for any larger set, say $\theta_{1}, \ldots, \theta_{n}$ with $n \geq m$, but there does not seem to be any converse in general: if we know a simultaneous approximation measure for $\theta_{1}, \ldots, \theta_{m+1}$, one may construct algebraic approximations to $\theta_{m+1}$ (using for instance the results of [19] Th. 3.2, [20] Th. 1.1, [10] Th. 2), but these may be lacunary and do not suffice to deduce a simultaneous approximation measure for $\theta_{1}, \ldots, \theta_{m}$.

Definition. Given two positive valued functions $\psi_{1}$ and $\psi_{2}$, let us write

$$
\psi_{1}(D, \mu) \prec \psi_{2}(D, \mu)
$$

if, for any $c>0$, there exist sequences $\left(D_{\nu}\right)_{\nu \geq 0}$ and $\left(\mu_{\nu}\right)_{\nu \geq 0}$ satisfying

$$
c \leq D_{\nu} \leq \mu_{\nu}, \quad D_{\nu} \leq D_{\nu+1} \leq 2 D_{\nu}, \quad \mu_{\nu} \leq \mu_{\nu+1} \leq 2 \mu_{\nu} \quad(\nu \geq 0),
$$

the sequence $\left(\mu_{\nu}\right)_{\nu \geq 0}$ is unbounded and

$$
\lim _{\nu \rightarrow \infty} \frac{\psi_{1}\left(D_{\nu}, \mu_{\nu}\right)}{\psi_{2}\left(D_{\nu}, \mu_{\nu}\right)}=0
$$

According to these definitions, Conjecture 1 means that a $m$-uple $\left(\theta_{1}, \ldots, \theta_{m}\right)$, for which a simultaneous approximation measure $\psi(D, \mu)$ satisfies

$$
\psi(D, \mu) \prec D^{1 / k} \mu
$$

generates a field of transcendence degree $\geq[k]+1$.

Conjecture 1 is likely to be the strongest possible result in this direction. We discuss this matter again in $\S 5$.

\section{Simultaneous Diophantine Approximation: a Conjecture}

Here is our second main Conjecture.

Conjecture 2. For each positive integer $n$ there exists a positive constant $c(n)$ with the following property. Let $\lambda_{1}, \ldots, \lambda_{n}$ be $\mathbb{Q}$-linearly independent logarithms of algebraic numbers and $\beta_{1}, \ldots, \beta_{n}$ be algebraic numbers. Define $\alpha_{i}=e^{\lambda_{i}} \quad(1 \leq i \leq$ n) and

$$
\underline{\gamma}=\left(\alpha_{1}, \ldots, \alpha_{n}, \beta_{1}, \ldots, \beta_{n}\right)
$$


Let $D$ be a positive integer and $\mu \geq D$ a real number satisfying

$$
[\mathbb{Q}(\underline{\gamma}): \mathbb{Q}] \leq D, \quad \mu(\underline{\gamma}) \leq \mu
$$

and

$$
\max _{1 \leq i \leq n}\left|\lambda_{i}\right| \leq \mu
$$

Then

$$
\sum_{i=1}^{n}\left|\lambda_{i}-\beta_{i}\right| \geq \exp \left\{-c(n) D^{1 / n} \mu\right\} .
$$

In fact for our purpose here it would be sufficient to get, for $n \geq 2$, an estimate of the form

$$
\sum_{i=1}^{n}\left|\lambda_{i}-\beta_{i}\right| \geq \exp \left\{-c(n) D^{\eta} \mu\right\}
$$

for some $\eta$ in the range

$$
0<\eta<\frac{1}{n-1}
$$

We now derive several consequences of Conjectures 1 and 2 together.

\subsection{Schanuel's Conjecture Under a Technical Hypothesis}

Assuming Conjectures 1 and 2, one deduces a special case of Schanuel's Conjecture, where the numbers $x_{1}, \ldots, x_{n}$ satisfy a so-called "technical hypothesis", which is the following measure of linear independence:

Definition. A n-uple $\left(x_{1}, \ldots, x_{n}\right)$ of complex numbers satisfies a linear independence measure condition if for any $\epsilon>0$ there exists $S_{0}>0$ such that, for any $S \geq S_{0}$ and any $n$-uple $\left(s_{1}, \ldots, s_{n}\right) \in \mathbb{Z}^{n}$ satisfying $0<\max _{1 \leq j \leq n}\left|s_{j}\right| \leq S$, we have

$$
\left|s_{1} x_{1}+\cdots+s_{n} x_{n}\right| \geq e^{-S^{\epsilon}} .
$$

Remark. This linear independence measure condition is (a weak form of) the socalled "Diophantine Condition" which occurs in the theory of dynamical systems. Such an assumption is not wanted for transcendence or algebraic independence results, but it cannot be avoided for quantitative statements (measures of transcendence, of approximation or of algebraic independence). In fact the approach we suggest here should also lead to such quantitative refinements, and from this point of view the technical condition is not unnatural. One may remark however that [19] completely avoids any linear independence measure condition. For large transcendence degree, removing such an assumption is still an open problem ([3], Chap. 6, § 4.3, p. 298; [24] § 3.2; [13]).

We now prove: 
Proposition 1. Let $\left(x_{1}, \ldots, x_{n}\right)$ be a n-uple of complex numbers which satisfies a linear independence measure condition. If Conjecture 2 holds, then there exists a constant $c>0$ such that the function

$$
\psi(D, \mu)=c D^{1 / n} \mu
$$

is a simultaneous approximation measure for the $2 n$ numbers

$$
x_{1}, \ldots, x_{n}, e^{x_{1}}, \ldots, e^{x_{n}} .
$$

Proof. Let $\gamma=\left(\alpha_{1}, \ldots, \alpha_{n}, \beta_{1}, \ldots, \beta_{n}\right)$ be a $2 n$-uple of algebraic numbers in a field of degree $\leq D$, and let $\mu \geq D$ satisfy $\mu(\underline{\gamma}) \leq \mu$. We assume that $D$ is sufficiently large. Assume also

$$
\sum_{i=1}^{n}\left(\left|x_{i}-\beta_{i}\right|+\left|e^{x_{i}}-\alpha_{i}\right|\right) \leq \exp \left\{-c_{1} D^{1 / n} \mu\right\}
$$

for some sufficiently large constant $c_{1}$ (depending only on $x_{1}, \ldots, x_{n}$ ). For $1 \leq i \leq$ $n$, we choose the logarithm $\lambda_{i}$ of $\alpha_{i}$ so that

$$
\sum_{i=1}^{n}\left|\lambda_{i}-\beta_{i}\right| \leq \exp \left\{-c_{2} D^{1 / n} \mu\right\} .
$$

From Conjecture 2 we deduce that the numbers $\lambda_{1}, \ldots, \lambda_{n}$ are linearly dependent over $\mathbb{Q}$. Therefore (see for instance $[26]$ ) there is a non trivial linear relation

$$
s_{1} \lambda_{1}+\cdots+s_{n} \lambda_{n}=0
$$

for some $\left(s_{1}, \ldots, s_{n}\right) \in \mathbb{Z}^{n}$ which satisfies

$$
0<\max _{1 \leq i \leq n}\left|s_{i}\right| \leq \mu^{c_{3}} .
$$

We deduce

$$
\left|s_{1} x_{1}+\cdots+s_{n} x_{n}\right| \leq \exp \left\{-c_{4} D^{1 / n} \mu\right\}
$$

and this is not compatible with the linear independence measure condition.

Since the exponent $\eta=1 / n$ of $D$ in the function $\psi$ of Proposition 1 satisfies $\eta<1 /(n-1)$, we deduce:

Corollary. Let $\left(x_{1}, \ldots, x_{n}\right)$ be a n-uple of complex numbers which satisfies a linear independence measure condition. Assume Conjectures 1 and 2 hold. Then the transcendence degree of the field

$$
\mathbb{Q}\left(x_{1}, \ldots, x_{n}, e^{x_{1}}, \ldots, e^{x_{n}}\right)
$$

is at least $n$.

We consider some examples. 


\subsection{First Example Involving Several Logarithms of Algebraic Numbers}

The Conjecture on algebraic independence of logarithms of algebraic numbers follows from Conjectures 1 and 2, and so does Lindemann-Weierstraß' Theorem. More generally, these two Conjectures 1 and 2 imply the following statement (which is an open problem):

- (?) Let $\lambda_{1}, \ldots, \lambda_{n}$ be $\mathbb{Q}$-linearly independent logarithms of algebraic numbers and $\beta_{1}, \ldots, \beta_{m}$ be algebraic numbers such that $1, \beta_{1}, \ldots, \beta_{m}$ are $\mathbb{Q}$ linearly independent. Then the $(n+1)(m+1)$ numbers

$$
e, \lambda_{i}, e^{\beta_{j}}, e^{\lambda_{i} \beta_{j}} \quad(1 \leq i \leq n, 1 \leq j \leq m)
$$

are algebraically independent.

Indeed, according to the Corollary, it is sufficient to check that the $(n+1)(m+1)$ uple

$$
1, \lambda_{i}, \beta_{j}, \lambda_{i} \beta_{j} \quad(1 \leq i \leq n, 1 \leq j \leq m)
$$

satisfies a linear independence measure condition. In fact, thanks to A. Baker, much stronger measures of linear independence for $1, \lambda_{1}, \ldots, \lambda_{n}$ over the field of algebraic numbers are known (see for instance [26]).

As an example, the algebraic independence of the four numbers $e, \pi, e^{\pi}$ and $e^{i}$ follows (take $n=m=1, \lambda_{1}=i \pi, \beta_{1}=i$ ).

The (open) problem of Gel'fond-Schneider ([3], p. 259; [7] $\S 2$, Conjecture 4; [24] Conjecture 1.2; [13]) is the following special case (take $n=1, m=d-1$, $\left.\beta_{j}=\beta^{j}\right)$ :

- (?) Let $\log \alpha$ be a non zero logarithm of an algebraic number and $\beta$ an algebraic number of degree $d$. Then the $d-1$ numbers

$$
\alpha^{\beta}, \ldots, \alpha^{\beta^{d-1}}
$$

are algebraically independent.

\subsection{Second Example Involving Powers of a Logarithm}

Another consequence of Conjectures 1 and 2 is:

- (?) Let $\lambda$ be a non zero logarithm of an algebraic number and let $\beta_{1}, \ldots, \beta_{m}$ be algebraic numbers such that $1, \beta_{1}, \ldots, \beta_{m}$ are $\mathbb{Q}$-linearly independent. Then the numbers

$$
e, \lambda, e^{\lambda^{2}}, e^{\lambda^{3}}, \ldots, \quad e^{\lambda^{k} \beta_{j}} \quad(k \geq 0,1 \leq j \leq m)
$$

are algebraically independent. 
Indeed, for any $n \geq 1$, the $(n+1)(m+1)$-uple

$$
\lambda^{k}, \lambda^{k} \beta_{j} \quad(0 \leq k \leq n, 1 \leq j \leq m)
$$

of complex numbers satisfies a linear independence measure condition.

For instance the algebraic independence of the numbers $e, \pi, e^{\pi}, e^{\pi^{2}}, \ldots, e^{\pi^{k}}$ follows (take $m=1, \lambda=i \pi, \beta_{1}=i$ ).

Remark. The algebraic independence of $2^{\log 2}$ and $e^{\pi}$ for instance (and more generally of numbers of the form $\lambda_{i}, e^{\lambda_{i}^{k} \beta_{j}}$ ) does not seem to follow from this approach. Henceforth it would be worthwhile to enforce Conjecture 1 so that one could deduce not only a lower bound for a transcendence degree, but also a quantitative refinement of this statement (e.g. a measure of algebraic independence). As pointed out above, the corresponding refinement of Corollary in $\S 3.1$ would then not be true without the linear independence measure condition.

\section{Simultaneous Diophantine Approximation: Results}

Under the hypotheses of Conjecture 2, only the weaker estimate

$$
\sum_{i=1}^{n}\left|\lambda_{i}-\beta_{i}\right| \geq \exp \left\{-c(n) \mu(\mu+D \log D)^{1+(1 / n)}\right\}
$$

(where $c(n)$ depends only on $n$ ) is known.

This lower bound is not optimal (as far as one expects!), and it does not include all what is known on this topic.

\subsection{Linear Algebraic Groups}

So far, all known partial results in direction of Conjecture 2 rest on the following general setup.

For $d_{0} \geq 0$ and $d_{1} \geq 1$, consider the commutative linear algebraic group $G=$ $\mathbb{G}_{\mathrm{a}}^{d_{0}} \times \mathbb{G}_{\mathrm{m}}^{d_{1}}$ of dimension $d=d_{0}+d_{1}$. The group of algebraic points of $G$ is $G(\overline{\mathbb{Q}})=\overline{\mathbb{Q}}^{d_{0}} \times\left(\overline{\mathbb{Q}}^{\times}\right)^{d_{1}}$ and the exponential map $\exp _{G}$ of $G(\mathbb{C})$ can be written

$$
\begin{array}{ccr}
\mathbb{C}^{d} & \longrightarrow & G(\mathbb{C})=\mathbb{C}^{d_{0}} \times\left(\mathbb{C}^{\times}\right)^{d_{1}} \\
\left(z_{1}, \ldots, z_{d}\right) & \longmapsto & \left(z_{1}, \ldots, z_{d_{0}}, e^{z_{d_{0}+1}}, \ldots, e^{z_{d}}\right)
\end{array}
$$

Let $W \subset \overline{\mathbb{Q}}^{d}$ be a vector space of dimension $m$ over $\mathbb{C}$ and $Y=\mathbb{Z} y_{1}+\ldots+\mathbb{Z} y_{\ell} \subset \mathbb{C}^{d}$ a subgroup of rank $\ell$ over $\mathbb{Z}$ such that $\exp _{G}(Y) \subset G(\overline{\mathbb{Q}})$ :

$$
y_{j}=\left(\beta_{1 j}, \ldots, \beta_{d_{0} j}, \lambda_{1 j}, \ldots, \lambda_{d_{1} j}\right) \in \overline{\mathbb{Q}}^{d_{0}} \times \mathcal{L}^{d_{1}},
$$


where

$$
\mathcal{L}=\exp ^{-1}\left(\overline{\mathbb{Q}}^{\times}\right)=\left\{\log \alpha ; \alpha \in \overline{\mathbb{Q}}^{\times}\right\}
$$

is the $\mathbb{Q}$-vector space of logarithms of algebraic numbers.

Under these circumstances, the main transcendence result is a lower bound for the dimension of the subspace $V$ spanned by $W$ and $Y$ in $\mathbb{C}^{d}$. Under suitable assumptions, one reaches:

$$
\operatorname{dim}_{\mathbb{C}} V \geq \frac{\ell d+m d_{1}}{\ell+d_{1}}
$$

What is needed here is an effective version (see [26] for more details).

\subsection{Two Estimates}

Here are two estimates which include many (but not all) Diophantine approximation results related to the exponential function (here, we restrict ourself to the one variable case, that is to one parameter subgroups of linear algebraic groups; see for instance [26] for further estimates).

We denote by $m$ and $n$ two positive integers and by $\alpha_{i j}(1 \leq i \leq m, 0 \leq j \leq n)$ non zero algebraic numbers in a number field $K$ of degree $\leq D$. For $1 \leq i \leq m$ and $0 \leq j \leq n$, let $\lambda_{i j}$ be any complex logarithm of $\alpha_{i j}$ : this means $e^{\lambda_{i j}}=\alpha_{i j}$. Let $A$ and $E$ be positive real numbers satisfying

$$
\mathrm{h}\left(\alpha_{i j}\right) \leq \log A, \quad\left|\lambda_{i j}\right| \leq \frac{D}{E} \log A \quad \text { and } \quad 1 \leq \log E \leq D \log A
$$

For $1 \leq i \leq m$ we write $\alpha_{i}$ and $\lambda_{i}$ in place of $\alpha_{i 0}$ and $\lambda_{i 0}$ respectively (these numbers occur only in Theorem 1 , not in Theorem 2). Also we use the absolute logarithmic height $\mathrm{h}$ on the projective spaces $\mathbb{P}_{n}$ (see for instance [26] Chap. 3).

We need some independence condition on these $\lambda_{i j}$. We introduce the following definition: given a positive real number $U$, we shall say that the $m \times n$ matrix $\left(\lambda_{i j}\right)_{\substack{1 \leq i \leq m \\ 1 \leq j \leq n}}$ satisfies the linear independence condition for $U$ if, for any $\underline{t} \in \mathbb{Z}^{m} \backslash\{0\}$ and any $\underline{s} \in \mathbb{Z}^{n} \backslash\{0\}$ with $\max _{1 \leq i \leq m}\left|t_{i}\right| \leq U$ and $\max _{1 \leq j \leq n}\left|s_{j}\right| \leq U$,

$$
\sum_{i=1}^{m} \sum_{j=1}^{n} t_{i} s_{j} \lambda_{i j} \neq 0 .
$$

Theorem 1. Define

$$
c_{1}=2^{32} m^{4} n^{2}(2 m)^{m / n} .
$$

Let $\beta_{1}, \ldots, \beta_{n}$ be algebraic numbers in the number field $K$ and let $B$ be a positive real number satisfying

$$
B \geq e, \quad B \geq D, \quad B \geq D \log A \quad \text { and } \quad \mathrm{h}\left(1: \beta_{1}: \cdots: \beta_{n}\right) \leq \log B .
$$


Define

$$
U_{1}^{m n}=D^{(m+1)(n+1)}(\log B)^{n+1}(\log A)^{m(n+1)}(\log E)^{-m-n-1}
$$

and assume that the $m \times(n+1)$ matrix $\left(\lambda_{i j}\right)_{\substack{1 \leq i \leq m \\ 0 \leq j \leq n}}$ satisfies the linear independence condition for $\left(c_{1} U_{1}\right)^{2}$. Assume further

$$
\log E \leq D \log B \leq U_{1} .
$$

Then

$$
\sum_{i=1}^{m} \sum_{j=1}^{n}\left|\lambda_{i j}-\beta_{j} \lambda_{i}\right| \geq e^{-c_{1} U_{1}}
$$

Theorem 2. Define

$$
c_{2}=2^{23} m^{3} n^{2}(2 m)^{m / n} .
$$

Let $\beta_{1}, \ldots, \beta_{n}, \beta_{1}^{\prime}, \ldots, \beta_{m}^{\prime}$ be algebraic numbers in $K$. Let $B$ be a positive real number satisfying the following conditions:

$$
\begin{gathered}
B \geq e, \quad B \geq D \log D, \quad B \geq D \log A \\
\mathrm{~h}\left(1: \beta_{1}: \cdots: \beta_{n}\right) \leq \log B \quad \text { and } \mathrm{h}\left(1: \beta_{1}^{\prime}: \cdots: \beta_{m}^{\prime}\right) \leq \log B .
\end{gathered}
$$

Define

$$
U_{2}^{m n}=D^{m n+m+n}(\log B)^{n+m}(\log A)^{m n}(\log E)^{-m-n}
$$

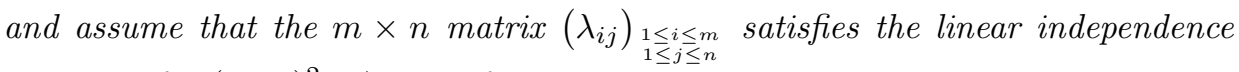
condition for $\left(c_{2} U_{2}\right)^{2}$. Assume further

$$
\log E \leq D \log B \leq U_{2} .
$$

Then

$$
\sum_{i=1}^{m} \sum_{j=1}^{n}\left|\lambda_{i j}-\beta_{j} \beta_{i}^{\prime}\right| \geq e^{-c_{2} U_{2}} .
$$

We now give several examples of these estimates. Further slightly sharper estimates can be found in [20] and [26].

\subsection{Consequences of Theorem 2}

The above mentioned estimate

$$
\sum_{i=1}^{n}\left|\lambda_{i}-\beta_{i}\right| \geq \exp \left\{-c(n) \mu(\mu+D \log D)^{1+(1 / n)}\right\}
$$

(under the hypotheses of Conjecture 2) follows from Theorem 2 by taking $m=1$, $\beta_{1}^{\prime}=1, B=n D^{2} e^{\mu / D}, A=e^{e \mu / D}, E=e$. 
Refinements are available in special cases. Here is a small sample.

\subsubsection{On Schanuel's Conjecture}

- Let $\left(x_{1}, \ldots, x_{n}\right)$ be a $n$-uple of complex numbers which satisfies a linear independence measure condition. Then a simultaneous approximation measure for the $2 n$ numbers

$$
x_{1}, \ldots, x_{n}, e^{x_{1}}, \ldots, e^{x_{n}}
$$

is

$$
\psi(D, \mu)=c \mu(\mu+D \log D)^{1+(1 / n)}(\log \mu)^{-1-(1 / n)},
$$

where $c$ depends only on $x_{1}, \ldots, x_{n}$.

One deduces this estimate from Theorem 2 by taking $m=1, B=\left(D e^{\mu / D}\right)^{c}$, $A=e^{\mu / D}, E=c^{\prime} \mu$ with suitable constants $c$ and $c^{\prime}$.

\subsubsection{Logarithms of Algebraic Numbers}

- Let $\lambda_{1}, \ldots, \lambda_{n}$ be $\mathbb{Q}$-linearly independent logarithms of algebraic numbers. There exists a positive number $c$ depending only on $\lambda_{1}, \ldots, \lambda_{n}$ such that a simultaneous approximation measure for the $n$-uple $\left(\lambda_{1}, \ldots, \lambda_{n}\right)$ is

$$
c D(\mu+D \log D)^{1+(1 / n)}(\log D)^{-1-(1 / n)} .
$$

One applies again Theorem 2 with $m=1, B=\left(D e^{\mu / D}\right)^{c_{1}}, A=c_{2}, E=c_{3} D$.

One should note here that another estimate (which is better when $\mu /(D \log D)$ is large) is due to N.I. Feldman [3], Chap. 3, Th. 3.34, namely

$$
c D^{1+(1 / n)}(\mu+D \log D)(\log D)^{-1} .
$$

However this is not yet sufficient to prove that the transcendence degree is $\geq 2$.

For further results on this topic, see [20].

\subsubsection{Lindemann-Weierstraß' Theorem}

- Let $\beta_{1}, \ldots, \beta_{n}$ be $\mathbb{Q}$-linearly independent algebraic numbers. Then a simultaneous approximation measure for the $n$-uple $\left(e^{\beta_{1}}, \ldots, e^{\beta_{n}}\right)$ is

$$
c D^{1 / n} \mu(\log \mu+D \log D)(\log \mu)^{-1} .
$$

The idea is to take $A=e^{c \mu / D}, B=\left(D \mu^{1 / D}\right)^{c}, E=\mu / c$. However such a choice does not satisfy the assumption $B \geq D \log A$ of Theorem 2. Fortunately it is possible to remove this condition in the present special case; we refer once more to [20], $\S 8$ for further details.

Assuming Conjecture 1, this estimate implies the algebraic independence of these $n$ numbers $e^{\beta_{1}}, \ldots, e^{\beta_{n}}$ (Lindemann-Weierstraß' Theorem). 


\subsection{Consequences of Theorem 1}

Here is a partial result related with Gel'fond-Schneider's Problem.

- Let $\log \alpha$ be a non zero logarithm of an algebraic number and let $\beta$ be an algebraic number of degree $d \geq 2$. Then a simultaneous approximation measure for the $(d-1)$-uple $\left(\alpha^{\bar{\beta}}, \ldots, \alpha^{\beta^{d-1}}\right)$ is

$$
c\left(\frac{D \mu^{d}}{\log \mu}\right)^{1 /(d-1)},
$$

for some $c>0$ depending only on $\log \alpha$ and $\beta$.

In case $\mu=D$ the exponent of $D$ is

$$
\frac{d+1}{d-1}=1+\frac{2}{d-1}
$$

Assuming Conjecture 1, it follows that the transcendence degree $t$ of the field

$$
\mathbb{Q}\left(\alpha^{\beta}, \ldots, \alpha^{\beta^{d-1}}\right)
$$

satisfies

$$
t \geq\left[\frac{d+1}{2}\right]
$$

This result of algebraic independence has been proved and is in fact the sharpest known one on this topic so far. It is due to G. Diaz and includes previous results of A.O. Gel'fond, W.D. Brownawell, A. Smelev, G.V. Chudnovsky, P. Philippon and others (see [3] Chap. 6, Cor. 6.3; [13]; [24] Th. 3.4; [26]).

The proof of this estimate uses the special case $n=d-1, m=d, \beta_{j}=\beta^{j}$ $(1 \leq j \leq d-1)$ of Theorem 1, with $A=e^{\mu / D}, B=d D \mu, E=\mu$. More precisely, assume $\gamma_{i}$ is an algebraic approximation of $\alpha^{\beta^{i}}$ for $1 \leq i \leq d-1$. We define $\gamma_{0}=\alpha$, and by induction we define $\gamma_{d}, \gamma_{d+1}, \ldots, \gamma_{2 d-2}$ so that $\gamma_{i}$ is an algebraic approximation of $\alpha^{\beta^{i}}$ for $0 \leq i \leq 2 d-2$. For $0 \leq i \leq 2 d-2$ we select the logarithm $\lambda_{i}$ of $\gamma_{i}$ which is close to $\beta^{i} \log \alpha$, and we put

$$
\lambda_{i j}=\lambda_{i+j-1} \quad \text { for } \quad 1 \leq i \leq d, 1 \leq j \leq d-1 .
$$

\subsection{Further Estimates}

Here are a few examples of further simultaneous approximation measures which follow from certain refinements of Theorems 1 and 2 (see [26]). 
4.5.1. On $\pi$ and $e^{\pi}$ From Theorem 2 with $m=n=2$, one deduces that there exists an absolute constant $c>0$ such that

$$
c(\mu+D \log D)^{2}(\log \mu)^{-1}
$$

is a simultaneous approximation measure for the two numbers $\pi$ and $e^{\pi}$. This estimate can be slightly refined, and the best known such measure is

$$
c D^{1 / 2} \mu(\mu+D \log D)^{1 / 2}(\log \mu)^{-1 / 2} .
$$

This is not strong enough to imply the algebraic independence of these two numbers! In fact for any quadratic number $\beta$ (in place of $i$ ) and any non zero logarithm of an algebraic number $\lambda$ (in place of $i \pi$ ) there exists a positive constant $c>0$ (depending on $\beta$ and $\lambda$ ) such that the same function

$$
c D^{1 / 2} \mu(\mu+D \log D)^{1 / 2}(\log \mu)^{-1 / 2} .
$$

is a simultaneous approximation measure for the two numbers $\lambda$ and $e^{\beta \lambda}([20]$ Th. 2.7). In general it is not yet proved that these two numbers $\lambda$ and $e^{\beta \lambda}$ are algebraically independent. is

A simultaneous approximation measure for the three numbers $\pi, e^{\pi}$ and $\Gamma(1 / 4)$

$$
c(\mu+D \log D)^{4 / 3}(\log \mu)
$$

for some absolute constant $c>0$. See [14] p. 328, [12], Th. 4, [15] Th. 3 and [16] Corollaire 5. The exponent $4 / 3$ is less than $3 / 2$; according to Conjecture 1 , one deduces Nesterenko's Theorem:

- The three numbers $\pi, e^{\pi}$ and $\Gamma(1 / 4)$ are algebraically independent.

The fact that a sharp simultaneous approximation measure is known for the three numbers $\pi, e^{\pi}$ and $\Gamma(1 / 4)$, but not for the two numbers $\pi$ and $e^{\pi}$, raises a natural question: assume that a $m$-uple $\left(\theta_{1}, \ldots, \theta_{m}\right)$ has a simultaneous approximation measure $\psi(D, \mu)$ satisfying $\psi(D, \mu) \prec D^{1 /(m-1)} \mu$. It follows from Conjecture 1 that the numbers $\theta_{1}, \ldots, \theta_{m}$ are algebraically independent, and therefore any subset of $\left\{\theta_{1}, \ldots, \theta_{m}\right\}$ consists of algebraically independent numbers. Can we deduce that for any $m^{\prime}$ in the range $1 \leq m^{\prime} \leq m$, there is a simultaneous approximation measure $\psi^{\prime}(D, \mu)$ for $\left(\theta_{1}, \ldots, \theta_{m^{\prime}}\right)$ which satisfies $\psi^{\prime} \prec D^{1 /\left(m^{\prime}-1\right)} \mu$ ?

As pointed out earlier, the answer is likely to be negative, and this calls for a modification of Conjecture 1 which would not have this drawback.

In [16] P. Philippon produces further Diophantine approximation results on these numbers, including the fact that $\Gamma(1 / 4)$ is not a Liouville number (but it is not yet proved that $e^{\pi}$ is not a Liouville number).

\subsubsection{On the Numbers $e$ and $\pi$}

- There exists an absolute constant $c>0$ such that

$$
c \mu^{1 / 2}(\mu+D \log D)\left(\mu+D^{2} \log D\right)^{1 / 2}(\log D)^{-3 / 2}
$$

is a simultaneous approximation measure for the two numbers $e$ and $\pi$. 
This is not sufficient to deduce the algebraic independence of these two numbers, and this is far from the expected $c D^{1 / 2} \mu$.

\subsubsection{On the Numbers $e, e^{e}$ and $e^{e^{2}}$}

- There exists an absolute constant $c>0$ such that

$$
\begin{aligned}
& \left|e-\gamma_{0}\right|+\left|e^{e}-\gamma_{1}\right|+\left|e^{e^{2}}-\gamma_{2}\right|> \\
& \quad \exp \left\{-c D^{2}\left(h_{0}+h_{1}+h_{2}\right)^{1 / 2}\left(h_{1}+h_{2}\right)^{1 / 2}\left(h_{0}+\log D\right)(\log D)^{-1}\right\}
\end{aligned}
$$

whenever $\gamma_{0}, \gamma_{1}, \gamma_{2}$ are algebraic numbers with

$$
h_{i}=\max \left\{1, \mathrm{~h}\left(\gamma_{i}\right)\right\} \quad(i=0,1,2) \quad \text { and } \quad D=\left[\mathbb{Q}\left(\gamma_{0}, \gamma_{1}, \gamma_{2}\right): \mathbb{Q}\right] .
$$

It follows (cf. [1], Cor. 3 and [23], Cor. 1) that one at least of the two numbers $e^{e}, e^{e^{2}}$ is transcendental. Indeed, the sequences $D_{\nu}=\nu$ and $\mu_{\nu}=\nu \log \nu$ (for instance) show that the function

$$
\psi(D, \mu)=c(D \mu)^{1 / 2}(\mu+D \log D)(\log D)^{-1}
$$

satisfies $\psi(D, \mu) \prec D \mu$, hence $\psi$ cannot be an approximation measure for $e$.

It is yet an open problem to prove that two at least of the three numbers $e, e^{e}$, $e^{e^{2}}$ are algebraically independent. The best known simultaneous approximation measure for these numbers is only $c \mu(\mu+\log D)(\log D)^{-1}$, which is not $\prec D \mu$.

\subsubsection{On the Numbers $\pi, e$ and $e^{\pi^{2}}$}

- There exists an absolute constant $c>0$ such that

$$
\begin{aligned}
&\left|\pi-\gamma_{0}\right|+\left|e-\gamma_{1}\right|+\left|e^{\pi^{2}}-\gamma_{2}\right|> \\
& \exp \left\{-c D^{2}\left(h_{0}+\log \left(D h_{1} h_{2}\right)\right) h_{1}^{1 / 2} h_{2}^{1 / 2}(\log D)^{-1}\right\}
\end{aligned}
$$

whenever $\gamma_{0}, \gamma_{1}, \gamma_{2}$ are algebraic numbers with

$$
h_{i}=\max \left\{1, \mathrm{~h}\left(\gamma_{i}\right)\right\} \quad(i=0,1,2)
$$

and

$$
D=\left[\mathbb{Q}\left(\gamma_{0}, \gamma_{1}, \gamma_{2}\right): \mathbb{Q}\right]
$$

We deduce that if the number $e^{\pi^{2}}$ is algebraic, then the function

$$
c(D \mu)^{1 / 2}(\mu+D \log D)(\log D)^{-1}
$$

(which we already met) is a simultaneous approximation measure for $\pi$ and $e$. Therefore (cf. [1], Cor. 4 and [23], Cor. 1) one at least of the two following statements is true:

(i) The numbers $\pi$ and $e$ are algebraically independent.

(ii) The number $e^{\pi^{2}}$ is transcendental.

Again the best known simultaneous approximation measure for the three numbers $\pi, e, e^{\pi^{2}}$ is $c \mu(\mu+D \log D)(\log D)^{-1}$, which is not sufficient to deduce that two of them are algebraically independent. 


\section{Distribution of Algebraic Points of Large Degree}

For each positive numbers $D$ and $\mu$ with $\mu \geq D$, let us define

$$
\overline{\mathbb{Q}}^{m}(D, \mu)=\left\{\underline{\gamma}=\left(\gamma_{1}, \ldots, \gamma_{m}\right) \in \overline{\mathbb{Q}}^{m} ;[\mathbb{Q}(\underline{\gamma}): \mathbb{Q}] \leq D, \mu(\underline{\gamma}) \leq \mu\right\} .
$$

That this is a finite subset of $\mathbb{C}^{m}$ is "a completely elementary result due to Northcott" ([6] Chap. II, Th. 2.2; see also [22] Lemma 7C).

Schanuel's counting Theorem ([6] Chap. II, Th. 2.5) provides an asymptotic formula for the number of points in a number field with bounded height, but here we do not fix the number field.

Conjecture 1 suggests to study the distribution of $\overline{\mathbb{Q}}^{m}(D, \mu)$ into $\mathbb{C}^{m}$. Our analysis is not sharp enough to make any difference with the smaller set where the condition

$$
[\mathbb{Q}(\underline{\gamma}): \mathbb{Q}] \max _{1 \leq i \leq m} \mathrm{~h}\left(\gamma_{i}\right) \leq \mu
$$

is replaced by an upper bound

$$
[\mathbb{Q}(\underline{\gamma}): \mathbb{Q}] \mathrm{h}\left(1: \gamma_{1}: \cdots: \gamma_{m}\right) \leq \mu
$$

involving the logarithmic height of the corresponding point in a projective space. Other variations are possible; for instance the condition on $\mu(\underline{\gamma})$ could be replaced by the upper bound

$$
\max _{1 \leq i \leq m} \mathrm{H}\left(\gamma_{i}\right) \leq e^{\mu}
$$

involving the usual height. A further interesting issue is how far the condition $\mu \geq D$ could be relaxed.

On the other hand it follows from D. Roy's recent paper [18] that the description which we are going to give does not hold for the subset

$$
\left\{\underline{\gamma} \in \overline{\mathbb{Q}}^{m}(D, \mu) ;[\mathbb{Q}(\underline{\gamma}): \mathbb{Q}]=D\right\}
$$

\subsection{The Subset $\overline{\mathbb{Q}}(D, \mu)$ of $\mathbb{C}$}

Let us start with the case $m=1$. A crude estimate for the number of points in $\overline{\mathbb{Q}}(D, \mu)$ is

$$
\log \operatorname{Card} \overline{\mathbb{Q}}(D, \mu) \simeq D \mu
$$

for sufficiently large $D$. We are interested with the distribution of $\overline{\mathbb{Q}}(D, \mu)$ in $\mathbb{C}$ when $D$ is sufficiently large.

We fix $\theta \in \mathbb{C}$. The case $\theta \in \overline{\mathbb{Q}}$ is not relevant, since then $\theta \in \overline{\mathbb{Q}}(D, \mu)$ for any sufficiently large $D$. So let us assume $\theta$ is transcendental. Let $D_{0}$ be sufficiently 
large (depending on $\theta$ ). For each $\kappa>0$, consider the set

$$
\mathcal{E}_{\kappa}(\theta)=\left\{(D, \mu) ; \mu \geq D \geq D_{0}, \min _{\gamma \in \overline{\mathbb{Q}}(D, \mu)}|\theta-\gamma| \leq \exp \{-\kappa D \mu\}\right\} .
$$

For $\kappa_{1} \leq \kappa_{2}$ we have $\mathcal{E}_{\kappa_{2}}(\theta) \subset \mathcal{E}_{\kappa_{1}}(\theta)$. For $\kappa>0$ the set $\mathcal{E}_{\kappa}(\theta)$ is empty if and only if $\kappa D \mu$ is an approximation measure for $\theta$.

We shall denote by

$$
\mathcal{E}_{\kappa}(\theta)^{c}=\left\{(D, \mu) ; \mu \geq D \geq D_{0},(D, \mu) \notin \mathcal{E}_{\kappa}(\theta)\right\}
$$

the complement set of $\mathcal{E}_{\kappa}(\theta)$. Then $\mathcal{E}_{\kappa}(\theta)^{c}$ is empty if and only if $\theta$ is at a distance $\leq \exp \{-\kappa D \mu\}$ of an algebraic number $\gamma$ of degree $\leq D$ and logarithmic measure $\leq \mu$.

If the sets $\overline{\mathbb{Q}}(D, \mu)$ were "well" distributed in $\mathbb{C}$, then for sufficiently large $\kappa$, say $\kappa \geq \kappa_{2}$, the set $\mathcal{E}_{\kappa}(\theta)$ would be empty, while for sufficiently small $\kappa$, say $\kappa \leq \kappa_{1}$, the complement $\mathcal{E}_{\kappa}(\theta)^{c}$ of $\mathcal{E}_{\kappa}(\theta)$ would be empty.

One can construct transcendental numbers $\theta$ (of the form $\theta=2^{\xi}$, where $\xi$ is a Liouville number - see [26], Exercise 17.8) for which each of these two properties fails for an infinite sequence of pairs $(D, \mu)$ with unbounded $D$ and $\mu$. More precisely, if $\psi$ is an approximation measure for such a $\theta$, then for any $D_{0}>0$ there is a sequence $\left(D_{\nu}, \mu_{\nu}\right)_{\nu \geq 0}$ with $\mu_{\nu} \geq D_{\nu} \geq D_{0}, \mu_{\nu} \rightarrow \infty$ and

$$
\frac{\psi\left(D_{\nu}, \mu_{\nu}\right)}{D_{\nu} \mu_{\nu}} \rightarrow \infty ;
$$

moreover there is another sequence $\left(D_{\nu}^{\prime}, \mu_{\nu}^{\prime}\right)_{\nu \geq 0}$ with $\mu_{\nu}^{\prime} \geq D_{\nu}^{\prime} \geq D_{0}, \mu_{\nu}^{\prime} \rightarrow \infty$ such that the function

$$
\psi_{\theta}(D, \mu)=-\log \min _{\gamma \in \overline{\mathbb{Q}}(D, \mu)}|\theta-\gamma|
$$

satisfies

$$
\frac{\psi_{\theta}\left(D_{\nu}^{\prime}, \mu_{\nu}^{\prime}\right)}{D_{\nu}^{\prime} \mu_{\nu}^{\prime}} \rightarrow 0 .
$$

The work of Y. Bugeaud [2] includes the following statement:

- For almost all complex numbers $\theta$, there exist $D_{1}(\theta)$ and $\kappa_{1}$ such that the complementary set of $\mathcal{E}_{\kappa_{1}}(\theta)$ (defined with $D_{0}=D_{1}(\theta)$ ) is empty.

From Lemma 5 of [2] then follows:

- For almost all complex numbers $\theta$, there exist $D_{2}(\theta)$ and $\kappa_{2}$ such that the set $\mathcal{E}_{\kappa_{2}}(\theta)$ (defined with $D_{0}=D_{2}(\theta)$ ) is empty.

Let us quote also the following result of A. Baker and W.M. Schmidt (see (3.12), Chap. VIII $\S 3$ in [21]):

- For almost all $\theta \in \mathbb{C}$, for any $D>0$ and any $\epsilon>0$, there exists a constant $c(D, \theta, \epsilon)$ such that

$$
|\theta-\gamma| \geq c(D, \theta, \epsilon) \mathrm{H}(\gamma)^{-D-1-\epsilon}
$$


for any $\gamma \in \overline{\mathbb{Q}}$ of degree $\leq D$.

Now we need to consider all elements of $\mathbb{C} \backslash \overline{\mathbb{Q}}$, and not only almost all complex numbers.

We introduce a few definitions.

Definition. $A$ regular sequence $\left(D_{\nu}, \mu_{\nu}\right)_{\nu \geq 0}$ is a sequence of elements of $\mathbb{N} \times \mathbb{R}_{>0}$ which satisfies

$$
D_{\nu} \leq \mu_{\nu}, \quad D_{\nu} \leq D_{\nu+1} \leq 2 D_{\nu}, \quad \mu_{\nu} \leq \mu_{\nu+1} \leq 2 \mu_{\nu} \quad(\nu \geq 0)
$$

and such that the sequence $\left(\mu_{\nu}\right)_{\nu \geq 0}$ is unbounded.

Definition. A subset $\mathcal{E}$ of $\mathbb{N} \times \mathbb{R}_{>0}$ is thick if there exists $c>0$ such that, for any regular sequence $\left(D_{\nu}, \mu_{\nu}\right)_{\nu \geq 0}$ satisfying $D_{0} \geq c$, the set

$$
\left\{\nu \geq 0 ;\left(D_{\nu}, \mu_{\nu}\right) \in \mathcal{E}\right\}
$$

is infinite.

Obviously if $\mathcal{E}^{\prime}$ is thick and $\mathcal{E}^{\prime} \subset \mathcal{E}^{\prime \prime}$, then $\mathcal{E}^{\prime \prime}$ is also thick. On the other hand if $\mathcal{E}$ is thick, then for any $D_{0}>0$ the set

$$
\left\{(D, \mu) \in \mathcal{E} ; D \geq D_{0}\right\}
$$

again is thick. By the way, the set $\mathcal{E}_{\kappa}(\theta)$ depends on $D_{0}$, but the property either for $\mathcal{E}_{\kappa}(\theta)$ or for $\mathcal{E}_{\kappa}(\theta)^{c}$ to be thick does not depend on $D_{0}$.

Also this remark allows us to define:

Definition. A subset $\mathcal{E}$ of $\mathbb{N} \times \mathbb{R}_{>0}$ is thin if its complementary set

$$
\mathcal{E}^{c}=\left\{(D, \mu) \notin \mathcal{E} ; \mu \geq D \geq D_{0}\right\}
$$

is thick. (This does not depend on $D_{0}$.)

One deduces from Th. 3.2 of [19] (see also Proposition 2 below) that for any transcendental number $\theta$, there exists $\kappa_{1}>0$ such that $\mathcal{E}_{\kappa_{1}}(\theta)$ is thick (and then $\mathcal{E}_{\kappa}(\theta)$ is thick for any $\kappa$ in the range $\left.0<\kappa \leq \kappa_{1}\right)$.

As kindly pointed out to me by Y. Bugeaud, it follows from Liouville's inequality that for any $\theta \in \mathbb{C} \backslash \overline{\mathbb{Q}}$, there exists $\kappa_{2}>0$ such that, for $\kappa \geq \kappa_{2}$, the set $\mathcal{E}_{\kappa}(\theta)$ is thin.

A subset $\mathcal{E}$ of $\mathbb{N} \times \mathbb{R}_{>0}$ may be neither thick nor thin: an example is

$$
\{(D, \mu) ; D \equiv 0 \quad(\bmod 2), \mu \geq D\} .
$$

On the opposite, if $\varphi: \mathbb{N} \rightarrow \mathbb{N}$ is a mapping satisfying

$$
\varphi(n+1) \geq 2 \varphi(n) \text { for } n \geq 0,
$$

then the set

$$
\bigcup_{k \geq 0}\{(D, \mu) ; \varphi(2 k) \leq D<\varphi(2 k+1), \mu \geq D\}
$$

is at the same time thick and thin! 
Finally we notice that, given two positive valued functions $\psi_{1}$ and $\psi_{2}$ on $\mathbb{N} \times$ $\mathbb{R}_{>0}$, the condition $\psi_{1}(D, \mu) \prec \psi_{2}(D, \mu)$ can be written: for any $\epsilon>0$, the set

$$
\left\{(D, \mu) ; \psi_{1}(D, \mu)>\epsilon \psi_{2}(D, \mu)\right\}
$$

is not thick.

\subsection{The Subset $\overline{\mathbb{Q}}^{m}(D, \mu)$ of $\mathbb{C}^{m}$}

Let $m$ be a positive integer. To give an asymptotic formula for the number of elements in $\overline{\mathbb{Q}}^{m}(D, \mu)$ is an open problem (cf [22] Chap. I, $\S 7$, p. 27 - notice that in Schmidt's Lecture Note [22], the normalization of the absolute logarithmic height at the Archimedean places involves the Euclidean norm). For us the following easy (and rough) estimate

$$
\text { Card } \overline{\mathbb{Q}}(D, \mu) \leq \operatorname{Card} \overline{\mathbb{Q}}^{m}(D, \mu) \leq(\operatorname{Card} \overline{\mathbb{Q}}(D, \mu))^{m}
$$

will suffice. Therefore $\log$ Card $\overline{\mathbb{Q}}^{m}(D, \mu)$ is (up to a multiplicative constant) $D \mu$.

Again we are interested with the distribution of $\overline{\mathbb{Q}}^{m}(D, \mu)$ in $\mathbb{C}^{m}$ when $D$ is sufficiently large and $\mu \geq D$.

We first notice that each affine algebraic curve of $\mathbb{C}^{m}$, which is rational over $\mathbb{Q}$, contains plenty of elements of $\overline{\mathbb{Q}}^{m}(D, \mu)$ :

- For any algebraic curve $\mathcal{C} \subset \mathbb{C}^{m}$, rational over $\mathbb{Q}$, there exists a constant $\kappa>0$ such that, for any sufficiently large $D$ and for $\mu \geq D$,

$$
\log \operatorname{Card}\left(\mathcal{C} \cap \overline{\mathbb{Q}}^{m}(D, \mu)\right) \geq \kappa D \mu \text {. }
$$

Moreover, on such a curve, the points of $\overline{\mathbb{Q}}^{m}(D, \mu)$ have a distribution which is similar to the distribution of $\overline{\mathbb{Q}}(D, \mu)$ in $\mathbb{C}$. Indeed, the case $m=1$ of Conjecture 1 , which is proved in [19], Th. 3.1 - see also [20] Cor. 1.2 - can be stated as follows:

Proposition 2. Let $\mathcal{C}$ be an affine algebraic curve in $\mathbb{C}^{m}$, rational over $\mathbb{Q}$. For any $\theta \in \mathcal{C}, \theta \notin \overline{\mathbb{Q}}^{m}$, there exist $D_{0}>0$ and $\kappa>0$ such that the set

$$
\left\{(D, \mu) ; \mu \geq D \geq D_{0}, \min _{\underline{\gamma} \in \overline{\mathbb{Q}}^{m}(D, \mu) \cap \mathcal{C}}|\underline{\theta}-\underline{\gamma}| \leq \exp \{-\kappa D \mu\}\right\}
$$

is thick.

An explicit version of Proposition 2 (including a lower bound for the degree $[\mathbb{Q}(\underline{\gamma}): \mathbb{Q}])$ is Th. 1 of $[10]$.

The distribution of elements of $\overline{\mathbb{Q}}^{m}(D, \mu)$ on a fixed algebraic subvariety $\mathcal{V}$ of $\mathbb{C}^{m}$, defined over $\mathbb{Q}$, should follow a similar pattern, with the exponent 1 of $D$ replaced by $1 / t$, where $t$ is the dimension of $\mathcal{V}$. This explains the following 
notation: For each $\underline{\theta} \in \mathbb{C}^{m} \backslash \overline{\mathbb{Q}}^{m}$, for sufficiently large $D_{0}$ and for each $\kappa>0$, we introduce the set

$$
\mathcal{E}_{\kappa}(\underline{\theta})=\left\{(D, \mu) ; \mu \geq D \geq D_{0}, \min _{\underline{\gamma \in \overline{\mathbb{Q}}^{m}(D, \mu)}}|\underline{\theta}-\underline{\gamma}| \leq \exp \left\{-\kappa D^{1 / t} \mu\right\}\right\},
$$

where $t$ is the transcendence degree over $\mathbb{Q}$ of the field $\mathbb{Q}(\underline{\theta})$.

Conjecture 3. Let $m$ be a positive integer.

1) For any $\underline{\theta} \in \mathbb{C}^{m} \backslash \overline{\mathbb{Q}}^{m}$, there exist $\kappa_{1}>0$ and $\kappa_{2}>0$ such that the set $\mathcal{E}_{\kappa}(\underline{\theta})$ is thick for $\kappa \leq \kappa_{1}$ and thin for $\kappa \geq \kappa_{2}$.

2) For almost all $m$-uples $\underline{\theta}=\left(\theta_{1}, \ldots, \theta_{m}\right)$ of complex numbers, there exist $\kappa_{1}>0$ and $\kappa_{2}>0$ such that, for $\kappa \leq \kappa_{1}$, the set $\mathcal{E}_{\kappa}(\underline{\theta})^{c}$ is empty, while for $\kappa \geq \kappa_{2}$, the set $\mathcal{E}_{\kappa}(\underline{\theta})$ is empty. by

For each $\underline{\theta} \in \mathbb{C}^{m} \backslash \overline{\mathbb{Q}}^{m}$, for sufficiently large $D$ and for $\mu \geq D$, define $\kappa_{\theta}(D, \mu)$

$$
\min _{\gamma \in \overline{\mathbb{Q}}^{m}(D, \mu)}|\theta-\gamma|=\exp \left\{-\kappa_{\theta}(D, \mu) D^{1 / t} \mu\right\}
$$

where $t$ is the transcendence degree over $\mathbb{Q}$ of the field $\mathbb{Q}(\underline{\theta})$. Hence

$$
\kappa_{\theta}(D, \mu)=\sup \left\{\kappa>0 ;(D, \mu) \in \mathcal{E}_{\kappa}(\underline{\theta})\right\} .
$$

Conjecture 3 states that for "most" pairs $(D, \mu)$ with $\mu \geq D$, we have

$$
\kappa_{1} \leq \kappa_{\theta}(D, \mu) \leq \kappa_{2} .
$$

More precisely, part 1 ) of Conjecture 3 states that the sets

$$
\left\{(D, \mu) ; \mu \geq D \geq D_{0}, \kappa_{\theta}(D, \mu)<\kappa_{1}\right\}
$$

and

$$
\left\{(D, \mu) ; \mu \geq D \geq D_{0}, \kappa_{\theta}(D, \mu)>\kappa_{2}\right\}
$$

are thin, while part 2 ) replaces "thin" by "empty" for almost all $\underline{\theta} \in \mathbb{C}^{m}$.

The existence of $\kappa_{1}$ in part 1 ) of Conjecture 3 is nothing else than Conjecture 1. The existence of both $\kappa_{1}$ and $\kappa_{2}$ would yield a "criterion" (i.e. a necessary and sufficient condition) for a $m$-uple $\underline{\theta} \in \mathbb{C}^{m}$ to generate a field of transcendence degree $t$.

For part 2) of Conjecture 3, it is likely that a stronger statement holds with constants $\kappa_{1}$ and $\kappa_{2}$ depending only on $m$, but not on $\underline{\theta}$ (running over a subset of $\mathbb{C}^{m}$ of measure 1 ). On the other hand one cannot expect that 2) would hold for any $\underline{\theta} \in \mathbb{C}^{m} \backslash \overline{\mathbb{Q}}^{m}$ : there exist algebraically independent numbers which, for infinitely many $(D, \mu)$, have good approximations by elements of $\overline{\mathbb{Q}}^{m}(D, \mu)$ :

- Let $f: \mathbb{N} \rightarrow \mathbb{N}$ satisfy

$$
\lim _{D \rightarrow \infty} f(D) \rightarrow \infty
$$

There exists a set $\left\{\theta_{a} ; 0 \leq a \leq 1\right\}$ of algebraically independent numbers such that, for any $m \geq 1$ and any $\left(a_{1}, \ldots, a_{m}\right) \in \mathbb{R}^{m}$ with $0 \leq a_{i} \leq 1$, for 
infinitely many $D>0$ there exists $\underline{\gamma} \in \overline{\mathbb{Q}}^{m}(D, D)$ such that

$$
\sum_{i=1}^{m}\left|\theta_{a_{i}}-\gamma_{i}\right| \leq e^{-D f(D)} .
$$

For instance ([26] Exercise 17.12), if $\Phi: \mathbb{N} \rightarrow \mathbb{N}$ is an increasing function such that

$$
\Phi(n+1) \geq 2 \varphi\left(2^{n \Phi(n)}\right) \quad(n \geq 0),
$$

then the numbers

$$
\theta_{a}=2^{\xi_{a}} \quad(0 \leq a<1) \quad \text { with } \quad \xi_{a}=\sum_{n \geq 1}\left[n^{2 a}\right] 2^{-\Phi(n)}
$$

provide a solution.

\subsection{Approximation by Cycles}

In place of simultaneous approximation by algebraic numbers, one may also consider other similar questions. An easy one is related with "polynomial approximation": given a $m$-uple $\underline{\theta}=\left(\theta_{1}, \ldots, \theta_{m}\right)$ of complex numbers, one seeks a non zero polynomial $f \in \mathbb{Z}\left[X_{1}, \ldots, X_{m}\right]$ such that $|f(\underline{\theta})|$ is "small". The existence of such a polynomial follows from Dirichlet's box principle (see for instance [19] Lemme 3.8, [10] Lemme 1, as well as [25] and [26]).

Next one asks for several "independent" such polynomials; more precisely one considers Diophantine approximation by cycles. This program has been suggested by P. Philippon [14], who already achieved partial results for small transcendence degree [17].

Here is a result dealing with approximation by a point on a hypersurface, due to M. Laurent and D. Roy [11] Th. 2.3.

Theorem (Laurent-Roy). Let $\underline{\theta} \in \mathbb{C}^{m}$. Let $\left(D_{n}\right)_{n \geq 1}$ be a non-decreasing sequence of positive integers, and let $\left(h_{n}\right)_{n \geq 1}$ be a sequence of real numbers $\geq 1$ such that $\left(D_{n} h_{n}\right)_{n \geq 1}$ is non-decreasing and unbounded. Then, for infinitely many $n$, there exists a non zero polynomial $P \in \mathbb{Q}\left[X_{1}, \ldots, X_{m}\right]$ of degree $\leq D_{n}$ and height $\mathrm{h}(P) \leq h_{n}$ which admits at least one zero $\underline{\alpha} \in \mathbb{C}^{m}$ with

$$
\max _{1 \leq i \leq m}\left|\theta_{i}-\alpha_{i}\right| \leq \exp \left(-\frac{1}{8(m+1) !} D_{n-1}^{m+1} h_{n-1}\right) \text {. }
$$

In the case $m=1$, we get $\underline{\alpha} \in \overline{\mathbb{Q}}\left(D_{n-1}, D_{n-1} h_{n-1}\right)$, and we recover Proposition 2. For $m \geq 1$, this statement together with Proposition 2 can be seen as a first step of an inductive process towards Conjecture 1, but the constants relative to a curve in Th. 1 of [10] are not yet sharp enough to conclude in the case $m=2$. 


\subsection{Measures}

A related question deals with quantitative refinement to Schanuel's Conjecture. One may expect that such a precise conjectural statement would include "all" expected results of Diophantine approximation for complex numbers related to the exponential function. It may be important to state a sufficiently sharp estimate; for instance this may help in an inductive argument.

Conjecture 2 is a first step in this direction, but it deals only with approximation by algebraic numbers. One may consider further (conjectural) estimates for higher dimensional cycles (which should include also Conjecture 1.4 of [24], for instance). Chow forms ([3], Chap. 6, §3, [14], [15], [16], [17] and [11]) seem to be a convenient tool to formulate such a desired general estimate.

\subsection{Metric Theory — Extremal Manifolds}

We propose further open problems involving Lebesgue's measure.

Our first question is:

- Which functions $\psi: \mathbb{N} \times \mathbb{R}_{>0} \rightarrow \mathbb{R}_{>0}$ are simultaneous approximation measures for almost all $m$-uples $\underline{\theta} \in \mathbb{C}^{m}$ ?

The following interesting phenomenon occurs. Denote by $\Psi$ the set of mappings $\psi$ which are simultaneous approximation measures for almost all elements of $\mathbb{C}^{m}$. For each $\psi \in \Psi$, denote by $E_{\psi}$ the subset of $\underline{\theta}$ in $\mathbb{C}^{m}$ for which $\psi$ is a simultaneous approximation measure. By definition $E_{\psi}$ contains almost all complex numbers, but

$$
\bigcap_{\psi \in \Psi} E_{\psi}=\emptyset .
$$

From the point of view of Diophantine approximation, no $m$-uple in $\mathbb{C}^{m}$ behaves like almost all m-uples!

Our last question is related with the problem of existence of $\kappa_{1}$ in part 2) of Conjecture 3. A positive answer would mean that for almost all $\underline{\theta} \in \mathbb{C}^{m}$, there exist $D_{0}>0$ and $\kappa>0$ such that, for any $\mu \geq D \geq D_{0}$,

$$
\min _{\gamma \in \overline{\mathbb{Q}}^{m}(D, \mu)}|\underline{\theta}-\underline{\gamma}| \leq \exp \left\{-\kappa D^{1 / m} \mu\right\} .
$$

It would be interesting to investigate whether a similar estimate holds also for almost all elements on a given analytic subvariety of $\mathbb{C}^{m}$ ? The meaning of Conjecture 1 is that the answer should be positive for algebraic subvarieties.

Such a problem, but involving only rational approximation, has been studied by many a mathematician, including K. Mahler, V.G. Sprindžuk (who coined the words extremal manifolds), A. Baker, W.M. Schmidt, G.V. Chudnovsky, V. Bernik, V. Beresnevich, M. Dodson, G.A. Margulis, D. Kleinbock. Approximation by 
algebraic numbers of bounded degree has been also considered from a metrical point of view, but here our concern is with algebraic numbers of large degree.

For instance, consider the analytic subvariety $z_{n+i}=e^{z_{i}} \quad(1 \leq i \leq n)$ in $\mathbb{C}^{2 n}$. A strong form of Schanuel's Conjecture holds almost everywhere: for almost all $n$ uples $\left(x_{1}, \ldots, x_{n}\right) \in \mathbb{C}^{n}$, the $2 n$ numbers $x_{1}, \ldots, x_{n}, e^{x_{1}}, \ldots, e^{x_{n}}$ are algebraically independent. Hence it seems natural to conjecture:

Conjecture 4 . Let $n$ be a positive integer. For almost all $n$-uples $\left(x_{1}, \ldots, x_{n}\right)$ of complex numbers, there exists a constant $c>0$ such that

$$
c D^{1 /(2 n)} \mu
$$

is a simultaneous approximation measure for the $2 n$ numbers

$$
x_{1}, \ldots, x_{n}, e^{x_{1}}, \ldots, e^{x_{n}} .
$$

\section{References}

[1] Brownawell, W. D., The algebraic independence of certain numbers related by the exponential function. J. Number Theory 6 (1974), 22-31.

[2] Bugeaud, Y., Remarques sur l'approximation par des nombres algébriques. (Submitted).

[3] Fel'dman, N.I.; Nesterenko, Y.V., Number theory. IV. Transcendental Numbers. Encyclopaedia of Mathematical Sciences, 44. Springer-Verlag, Berlin, 1998.

[4] Gramain, F., Quelques résultats d'indépendance algébrique. Proc. Intern. Cong. Math., Vol. 1, (Berlin, 1998), 173-182, Documenta Mathematica (J. D. M. V.) 1998.

[5] Lang, S., Introduction to transcendental numbers. Addison-Wesley Publishing Co., Reading, Mass.-London-Don Mills, Ont. 1966.

[6] Lang, S., Number theory. III. Diophantine geometry. Encyclopaedia of Mathematical Sciences, 60. Springer-Verlag, Berlin, 1991. Corrected second printing: Survey of Diophantine Geometry; 1997.

[7] Laurent, M., New methods in algebraic independence. Number Theory-Diophantine, Computational and Algebraic Aspects, K. Györy, A. Pethő and V.T. Sòs, eds., Proc. Conf. Number Theory Eger 1996, W. de Gruyter, Berlin (1998), 311-330.

[8] Laurent, M., On the approximation of complex numbers by algebraic numbers. Lecture in Thessaloniki. June 1998, (to appear). 
[9] Laurent, M.; Roy, D., Criteria of algebraic independence with multiplicities and interpolation determinants. Trans. Amer. Math. Soc., (to appear).

[10] Laurent, M.; Roy, D., Sur l'approximation algébrique en degré de transcendance un. Annales Instit. Fourier, (to appear).

[11] Laurent, M.; Roy, D., Criteria of algebraic independence with multiplicities and approximation by hypersurfaces, (submitted).

[12] Nesterenko, Y.V., On the measure of algebraic independence of the values of Ramanujan functions. Trudy Mat. Inst. Steklov. 218 (1997), 299-334; English translation in Proceedings of the Steklov Institute of Mathematics, 218 (1997), 294-331.

[13] Nesterenko, Y.V.; Philippon, P., Eds, Introduction to Algebraic Independence Theory. Instructional Conference (CIRM Luminy 1997), (in preparation).

[14] Philippon, P., Une approche méthodique pour la transcendance et l'indépendance algébrique de valeurs de fonctions analytiques. J. Number Theory 64 (1997), no. 2, 291-338.

[15] Philippon, P., Indépendance algébrique et $K$-fonctions. J. reine angew. Math., 497 (1998), 1-15.

[16] Philippon, P., Mesures d'approximation de valeurs de fonctions analytiques. Acta Arith., (to appear).

[17] Philippon, P., Approximations algébriques des points dans les espaces projectifs. (Submitted).

[18] Roy, D., Approximation algébrique simultanée de nombres de Liouville. (Submitted).

[19] Roy, D.; Waldschmidt, M., Approximation diophantienne et indépendance algébrique de logarithmes. Ann. scient. Ec. Norm. Sup., 30 (1997), no 6, 753-796.

[20] Roy, D.; Waldschmidt, M., Simultaneous approximation and algebraic independence. The Ramanujan Journal, 1 Fasc. 4 (1997), 379-430.

[21] Schmidt, W. M., Diophantine approximation. Lecture Notes in Mathematics, 785. Springer, Berlin, 1980.

[22] Schmidt, W. M., Diophantine approximations and Diophantine equations. Lecture Notes in Mathematics, 1467. Springer-Verlag, Berlin, 1991.

[23] Waldschmidt, M., Solution du huitième problème de Schneider. J. Number Theory 5 (1973), 191-202. 
[24] Waldschmidt, M., Algebraic independence of transcendental numbers: a survey. Monograph on Number Theory, Indian National Science Academy, R.P. Bambah, V.C. Dumir and R.J. Hans Gill (eds), (to appear).

[25] Waldschmidt, M., From simultaneous approximations to algebraic independence. In Number theory and its applications, Cem Y. Yıldırım and Serguei Stepanov Ed., Lecture Notes in Pure and Applied Mathematics 204, Marcel Dekker Inc., 283-305.

[26] Waldschmidt, M., Diophantine Approximation on Linear Algebraic Groups. Transcendence Properties of the Exponential Function in Several Variables, (in preparation).

Address of the author:

Michel. Waldschmidt

Mathematiques, UFR 920

Université P. et M. Curie, P.B. 172

4, place Jussieu

F-75252 Paris Cedex 05

France

E-mail: miw@math.jussieu.fr 